\title{
Analysis of Type II radio burst relationship with CME driven shocks
}

\author{
"Raveesha K.H' ${ }^{1}$, Vedavathi $\mathbf{P}^{2}$, Vijayakumar H Doddamani ${ }^{3}$ \\ ${ }^{1}$ Department of Physics, CMR Institute of Technology, Bangalore -560037, India \\ ${ }^{2,3}$ Department of Physics, Bangalore university,Bengaluru-560056, India
}

Email : hod.physics@cmrit.ac.in

\begin{abstract}
Type II radio bursts are known to be the signatures of coronal shocks. In this paper we examine the relationship between 129 type II bursts in the frequency range $35-450 \mathrm{MHz}$ observed at Culgooora observatory during May 2002 - October 2015 and the associated CMEs. We apply Newkirk (1961) density model to determine the formation height of type IIs. We find that in 109/129 cases, type II bursts were preceded/ succeeded by CMEs. The CME associated type II events in which the CME height is above the type II burst source are categorized as group I events (91/129 cases). 91\% of the bursts in this group are also associated with flares and 58\% of these bursts originate during decaying phase of the flare. The correlation between CME speed and type II shock speed for limb events in this group is 0.33. The CME associated type IIs in which the CME height is below the type II source are categorized as group II (18/129 cases). CME driven shock could have been the exciter of these type II bursts.88\% of this group events are associated with flares and $62 \%$ of these bursts originate during the rising phase of the flare. The correlation between CME speed and type II shock speed for limb events in this group is 0.96. In 20/129 cases of our data set, type II bursts are not associated with CME and are categorized as group III. $90 \%$ of the bursts in this group are associated with flares. $77 \%$ of the bursts in the group are originating in the decaying phase of flares. Poor temporal association (9/69 cases) between type IIs and flares of X class during this period. Our results suggest that inspite of temporal association with metric type II bursts, majority of the CME driven shocks (84\%) are not successful in exciting type II bursts in $35-450 \mathrm{MHz}$ domain. The type II bursts temporally correlated with CMEs and likely to have been excited by CMEs (type II height > CME height) are originating during the rising phase of the flares in majority of the events. In case of type II bursts temporally correlated with CMEs supposedly not excited by the CMEs (type II height < CME height) , majority of them are originating in the decaying phase of flares.
\end{abstract}

Key words: Coronal Mass Ejection-shock-metric-observatory-flare-impulsive

\section{INTRODUCTION}

Solar type II radio bursts are known to be the signatures of magneto hydrodynamic (MHD) shocks propagating through the solar atmosphere. They are observed as slow drifting emission bands fundamental $(\mathrm{F})$ and harmonic $(\mathrm{H})$ with a frequency ratio of $\approx 1: 2$. The frequency drift from high to low frequencies (typically $0.5 \mathrm{MHz} / \mathrm{s}$ ) is a measure of the decrease of electron density $\left(N_{e}\right)$ with radial distance $\mathrm{r}$ in the solar atmosphere. The observed drift rate can be utilized to find velocity if the dependence of $N_{e}$ on $r$ is known. The characteristics description of solar type II bursts can be found in Nelson \& Melrose (1985), Mann et al. (1995), Aurass (1997), and Gopalswamy (2006). MHD shocks in the lower corona $\left(1-2 R_{o}\right)$ have been attributed to either solar flares or coronal mass ejections (CMEs) or combination of the two (Gopalswamy 2006; Mann \& Vr`snak 2007; Pick \& Vilmer 2008; Vr`snak \& Cliver 2008; Nindos et al. 2008). CMEs occurring at the solar limb can be seen in coronagraph images than those near the center of the solar disk (Cliver et al. 1999; Gopalswamy et al. 2001).

Results obtained using radio spectral observations of metric type II bursts indicate that they can be excited by CME driven shocks shocks (Lara et al. 2003; Cliver et al. 2004; Cho et al. 2005, 2007, 2008, 2011; Subramanian \& Ebenezer 2006; Mancuso 2007; Gopalswamy et al. 2009a; Liu et al. 2009).Many of these works were carried out without positional information on the type II bursts. Radio heliograph data carry positional information of the type II bursts. Hence the spatial relationship with CMEs and flares can be verified using them. Type II bursts with positional information have been reported by authors (Dulk 1970; Stewart et al. 1974a, 1974b; Nelson \& Robinson 1975; Kosugi 1976; Wagner \& MacQueen 1983; Gergely et al. 1983; Gary et al. 1984; Gopalswamy \& Kundu 1992; Maia et al. 2000; Magdaleni'c et al. 2010; Ramesh et al. 2010; Nindos et al. 2011). These works consisted few selective events. 
The relationship between type Shanghai for Science and Technel pgy bursts, flares, and coronal mass ejections (CMEs) is not clear. The relationship between metric type II bursts and CMEs is debatable question (Cliver et al. 2004; Vršnak \& Cliver 2008; Prakash et al. 2010), whereas for type II bursts at decameter and longer wavelengths there is a consensus that they are driven by CMEs (Cane et al. 1987; Gopalswamy et al. 2000). According to Vrsnak and Cliver (2008) the source of the coronal wave is clear in some events with starting frequencies well below $100 \mathrm{MHz}$, where the CME is accompanied by a weak flare. They observed that for Moreton-wave-associated type II bursts at higher frequencies (>300 MHz for the harmonic emission), both the CME and the flare are observed. In these events, the CME and the flare are tightly related and, particularly during the impulsive phase, motions of the flare plasma take place together with the CME motions, and both phenomena are potential candidates for producing shocks (Vršnak \& Cliver 2008; Magdaleni'c et al. 2012). The study of the relationship between type II bursts and CMEs can be improved by observations of propagating brightness fronts in the extreme ultraviolet (EUV), so-called EUV waves (Moses et al. 1997; Thompson et al. 1998).

Shock waves are an ubiquitous phenomenon in astrophysics. They accelerate electrons (Mann \& Classen 1995; Miteva \& Mann 2007; Schwartz et al. 2011) and ions (Thomsen et al. 1985; Sckopke 1995; Giacalone 2005).In the solar atmosphere, several kinds of shocks, which manifest as wave-like structures in images are implied in the radio dynamic spectra. Type II radio bursts, following the eruption of coronal mass ejections (CMEs), are a kind of plasma emission with a slow frequency drift in the radio dynamic spectrum, and are generally known to be a signature of coronal shocks (Wild 1950; Zheleznyakov 1970). It is known that most of the interplanetary shocks (within 1 AU) are CME-driven (Cane et al. 1987; Reiner et al. 2001a, 2001b). However, the origin of the metric type II radio bursts is still an open question; they can be generated either by CME-driven shocks (Cliver et al. 2004; Liu et al. 2009; Chen 2011; Cho et al. 2013) or by flare-caused blast waves (Leblanc et al. 2001; Magdalenić et al. 2008; Nindos et al. 2011).

Our present work utilizes a comparatively larger data set (129 events) in the frequency range $35 \mathrm{MHz}$ to $450 \mathrm{MHz}$. In this study we estimate the height of the CME driven shock at the onset of the type II radio bursts.129 events are categorized as Group I (91/129) representing events with CME height at the onset of type II is above the Type II formation height, Group II (18/129) indicating events for which CME height is below the type II formation height and group III (20/129) for type II events not temporally associated with CMEs. We assume that coronal shocks producing metric type II radio bursts are driven by CMEs whose formation height is less than that of type IIs. We calculate the CME height at the type II onset by CMEs extrapolated back to the start time of the type II bursts assuming a constant speed of the CMEs. This approximation is reasonable as acceleration effect will be negligible at high velocities. The kinematics of type II shock is determined by adopting suitable coronal density models. In fact, the real density distribution at the lower corona varies with time and location and the observed CME kinematics might be different at the height where the type II bursts occur since the flare impulsive phase is often associated with CME acceleration phase (Bemporad et al.2003; Guhathakurta \& Fisher 1998; Parenti et al. 2000; Zhang et al. 2001).

This paper is organized as follows. Data set is provided in Section 2, data analysis and results are presented in Section 3, followed by a conclusion in Section 4 .

\section{DATA SET}

In this work the type II radio data recorded by the Culgoora observatory (http://www.sws.bom.gov.au/Solar/2/5/1) for the period May 2002 to October 2015 is referred. The detailed description is available at this site. The CME data provided by Large Angle Spectrometric Coronograph (LASCO; Brueckner et al.1995) CME catalog http : // cdaw.gsfc.nasa.gov / CME_list /index.html; Yashiro et al.2004; Gopalswamy et al.2009b) on board the Solar Wind Heliospheric Observatory (SoHO) is used to examine whether the radio burst is associated with a CME.. CMEs occurring within 20-30 minutes preceding/succeeding type II bursts have been considered and in most of the cases the temporal association is within 15 minutes. We identified all those Type II radio events for which adequate CME data is available. Few type II events with unclear start time/ end time, insufficient data have not been considered. Our final data set consists of 144 type II bursts categorized as Group I, II \& III respectively are listed in table 1, $2 \& 3$. The columns in the table 1, 2 are : (1) type II date (2) start time (3) end time (4) drift rate (5) shock speed (6) associated flare peak time (7) flare location (8) associated CME detected time (9) CME height

type II $\quad$ start $\quad(10) \quad$ type II formation height $\quad$ (11) type II formation height minus CME height. The columns in table 3 are: (1) type II date (2) start time (3) end time (4) drift rate (5) shock speed (6) associated flare peak time (7) flare location (8) type II formation height. The columns in table 4 are similar to the previous table along with X flare details. We use the flare data recorded by the low energy channel $(0.5-4 \AA)$ of the Reuven Ramaty High Energy Solar Spectroscopic Imager (RHESSI; Lin et al.2002). Flares occurring within +/- 20-30 minutes from the onset of type II bursts are considered as associated flares. For most of the events in our data set, flares/CMEs are observed within 20 minutes from the type II event. Figure 1a shows the dynamic spectrum from Culgoora observatory recorded on 17/05/2012. The frequency range of the spectrometer is from $1800 \mathrm{MHz}$ to $18 \mathrm{MHz}$. The fundamental type II burst started from about $40 \mathrm{MHz}$ at $01: 32$ and drifted to $18 \mathrm{MHz}$ at $01: 40$. The mean frequency drift rate of the type II is about $0.20 \mathrm{MHz} / \mathrm{s}$ close to typical drift rate $(\sim 0.3 \mathrm{MHz} / \mathrm{s})$ of type IIs reported by Mann (1995). The harmonic band starts at around $80 \mathrm{MHz}$ at 01:32. As seen from the figure $1 \mathrm{~b}$, the early eruption of type II associated CME was observed by Large Angle and Spectrometric Coronagraph (LASCO $-\mathrm{C} 2 / \mathrm{C} 3$ ) onboard the Solar and Heliospheric Observatory (SOHO) at 01:48. The first appearance of the CME was at $3.63 \mathrm{R}_{\mathrm{o}}$ above the solar surface. The acceleration of the CME is $-51.8 \mathrm{~km} / \mathrm{s}^{2}$. The CME height at the onset of Type II 


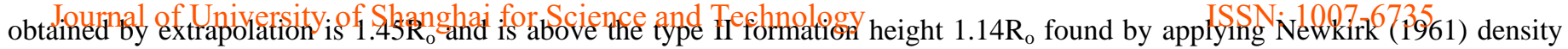
model. This CME is likely to have not excited the type II shown in figure 1a. Figure 1c shows the height -time plot of the leading edge of CME from 01:20 to 04:40.

Figure 1d shows RHESSI X ray flux in the $6-12 \mathrm{keV}, 12-25 \mathrm{keV}$ channels. The associated flare commences at 00:23 reaches the peak at 01:48 close to the type II burst onset time 01:32. In addition, figure 1e shows an $\mathrm{M}$ class flare observed by the Geostationary Operational Environmental Satellite (GOES) with onset at 00:28.

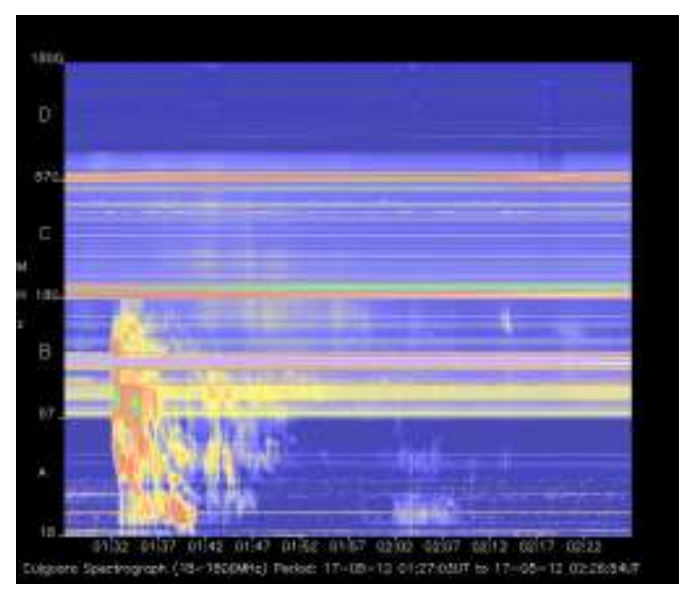

(a)

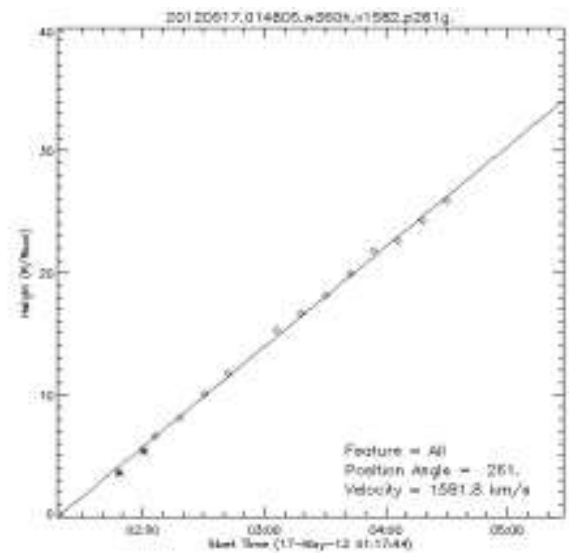

(c)

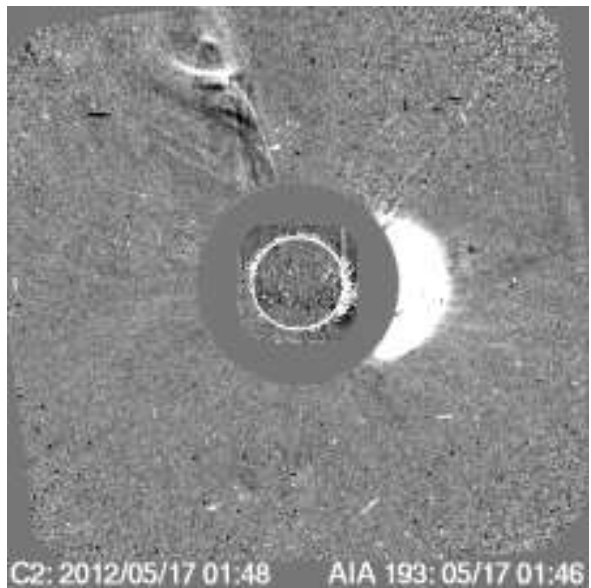

(b)

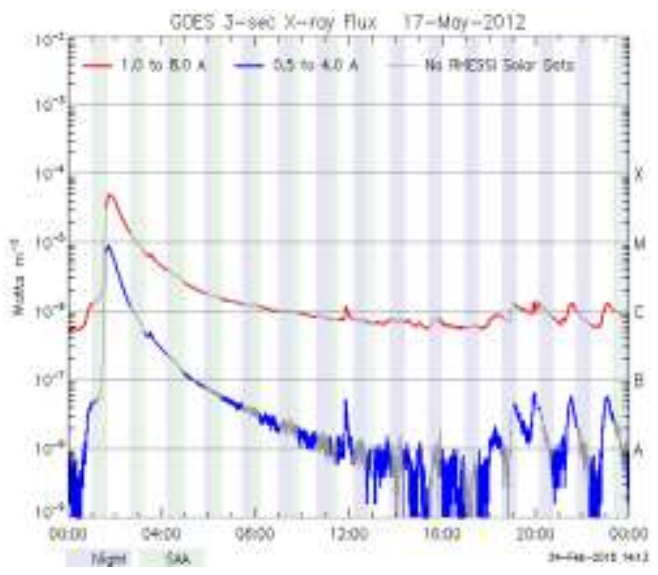

(d)

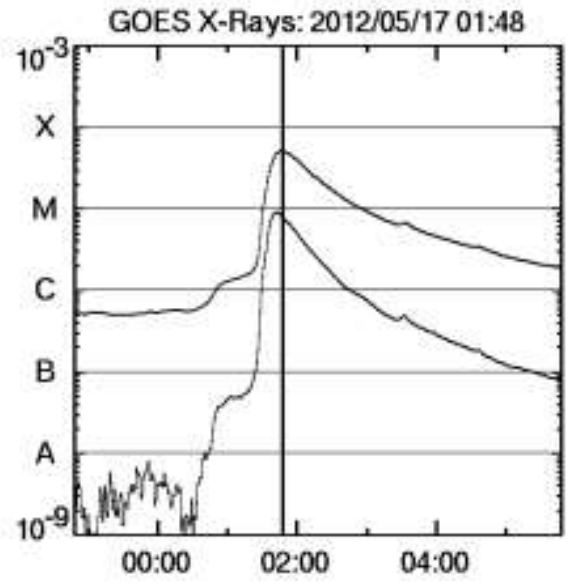

(e)

\section{Figure 1.}

(a) Dynamic spectrum of the type II radio burst observed by Culgoora observatory from 01:32-01:40 on 17/5/2012. The burst commences at $40 \mathrm{MHz}$ and drifts to $18 \mathrm{MHz}$. Weak band splitting is observed.

(b) The type II associated CME observed by LASCO on 17/5/2012 detected at 01:48 at 3.61 $\mathrm{R}_{\mathrm{o}}$ occurring 13 minutes after the onset of type II burst.

(c) Height -time plot of the leading edge of CME from 01:20 to 04:40 


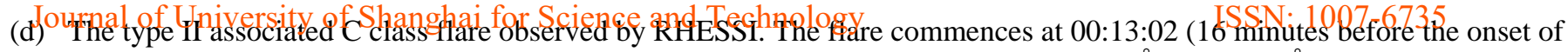
type II burst) and reaches peak at 01:41 before decaying at 07:26.X ray spectrum at 1-8 $\mathrm{A}$ and $0.5-4 \AA$ are shown.

(e) M class flare observed by the Geostationary Operational Environmental Satellite (GOES) with onset at 00:28.The peak time is 01:48 in agreement with RHESSI records.

\section{DATA ANALYSIS AND RESULTS}

\subsection{Type II - CME height relationship}

The starting frequencies of type II bursts represent the heights of the radio sources: a higher starting frequency indicates a radio source closer to the solar surface because the frequency of the type II emission is proportional to the square root of the plasma density and the plasma density decreases as a function of radial distance. Most of the density models may differ significantly from the real case and affect the height calculation. In previous studies, the most commonly used density models are Newkirk (1961) and Saito et al. (1977). Since the use of Newkirk (1961) density model to estimate the radio source speed is compatible with radial shock motion [58], we have used Newkirk (1961) density model to determine the formation of height of type II bursts. The Newkirk (1961) density model is given by

$\mathrm{N}_{\mathrm{e}}=4.2 \times 10^{4} \times 10^{4.32(\mathrm{Ro} / \mathrm{R})} \mathrm{cm}^{-3} \quad$ where $\mathrm{R}$ is the heliocentric distance and $\mathrm{R}_{\mathrm{o}}$ is the solar radius.

The CME height at the onset of type II is calculated by back extrapolation assuming constant speed of CMEs.

CME height (at the onset of type II) $=$ CME first appearance height $+/-\mathrm{V}_{\mathrm{CME}} * \mathrm{dt}$

Here dt is the start time difference between type II \& CME.

Table 1 provides details of 91 group I events ( type II height - CME height is negative).It is observed that the type II formation height is in the range of $1.005 \mathrm{R}_{\mathrm{o}}$ to $1.36 \mathrm{R}_{\mathrm{o}}$ for more than $83 \%$ of the events with an average of $1.16 \mathrm{R}_{\mathrm{o}}$ close to the results of Cunha-Silva et al.(2015). The average start and end frequency are $201.8 \mathrm{MHz}$ and $39.5 \mathrm{MHz}$ respectively with an average bandwidth of $163.34 \mathrm{MHz}$. The average drift rate is $0.24 \mathrm{MHz} / \mathrm{s}$ close to the typical drift rate $(\sim 0.3 \mathrm{MHz} / \mathrm{s})$ of type IIs reported by Mann et al.(1995). The average shock speed is $776 \mathrm{~km} / \mathrm{s}$ and the average duration is 10.7 minutes.

The CMEs observed in association with these 91 type II events are at heights above the type II formation heights (average height $3.59 \mathrm{R}_{\mathrm{o}}$ ). The average width and central position angle of these CMEs is $101.6 \mathrm{deg}$ and $203.5 \mathrm{deg}$.The average speed of these CMEs is $695 \mathrm{~km} / \mathrm{s}$ greater than typical speed of CMEs $(450 \mathrm{~km} / \mathrm{s})$. $65 \%$ of CMEs showed deceleration in this group with an average of $-5 \mathrm{~km} / \mathrm{s}^{2} .27 \%$ of them are halo CMEs. Poor correlation $(0.33)$ is observed between CME speed and type II shock speed for limb events supporting the argument that blast waves from flares could have excited these type IIs.

Flares are associated in $91 \%$ of the events and $58 \%$ of these bursts originate during decaying phase of the flare. The drift rates of type IIs associated with different classes of flares are studied for limb events (20/91) in this group. The average drift rate for type IIs accompanying $\mathrm{X}, \mathrm{C}$ and $\mathrm{M}$ class flares in this category is $0.27 \mathrm{MHz} / \mathrm{s}, 0.22 \mathrm{MHz} / \mathrm{s}$ and $0.17 \mathrm{MHz} / \mathrm{s}$ respectively. Higher drift rate for bursts associated with $\mathrm{X}$ class flares is possibly due to their higher energy. This probably leads to higher shock speed and corresponding higher Alfven speed. This possibly could be one of causes for the absence of association between Type II and X class flares. It may also be noted that drift rate corresponds to energy associated with the respective class of the flare.

Table 2 provides the details of 18 group II events (type II height - CME height is positive). It is observed that the type II formation height is in the range of $1.07 \mathrm{R}_{\mathrm{o}}$ to $1.29 \mathrm{R}_{\mathrm{o}}$ for more than $55 \%$ of the events with an average of $1.19 \mathrm{R}_{\mathrm{o}}$. The average start and end frequency are $194.8 \mathrm{MHz}$ and $36.3 \mathrm{MHz}$ respectively with an average bandwidth of $158.5 \mathrm{MHz}$.The average drift rate is $0.20 \mathrm{MHz} / \mathrm{s}$ slightly less than the typical drift rate $(\sim 0.3 \mathrm{MHz} / \mathrm{s})$ of type IIs reported by Mann et al. (1995). The average shock speed is $745 \mathrm{~km} / \mathrm{s}$ and the average duration is 11.14 minutes.

The CMEs observed in association with these 18 type II events are originating at a height lower than the type II formation heights (avg ht $1.19 \mathrm{R}_{\mathrm{o}}$ ). The average width and central position angle of these CMEs is $162 \mathrm{deg}$ and $211.8 \mathrm{deg}$. The average speed of these CMEs is $1101 \mathrm{~km} / \mathrm{s}$ which is almost 3 times greater than typical speed of CMEs $(450 \mathrm{~km} / \mathrm{s}) .74 \%$ of CMEs showed deceleration in this group with an average of $-15 \mathrm{~km} / \mathrm{s}^{2} .44 \%$ of them are halo CMEs. The strong correlation $(0.96)$ between CME speed and type II shock speed for limb events is noticed. This supports the view that these type IIs could have been excited by CME driven shocks.

Flares are associated in $88 \%$ of these events. Unlike group I events, $62 \%$ of these bursts originate during the rising phase of the flare. Average drift rate for type IIs accompanying $\mathrm{C}$ and $\mathrm{M}$ class flares in this category is $0.16 \mathrm{MHz} / \mathrm{s}, 0.20 \mathrm{MHz} / \mathrm{s}$ respectively.

The details of group III type II events not associated with CMEs are given in table 3. It is observed that the type II formation height is in the range of $1.11 \mathrm{R}_{\mathrm{o}}$ to $1.29 \mathrm{R}_{\mathrm{o}}$ for more than $63 \%$ of the events with an average of 1.24Ro.The average start frequency is $138 \mathrm{MHz}$ significantly lesser than group I and II events. The average drift rate is $0.26 \mathrm{MHz} / \mathrm{s}$. The average shock speed is $645 \mathrm{~km} / \mathrm{s}$ which is lesser than the group $1 \& 2$ bursts. The average duration is 8.16 minutes. Flares are present in $90 \%$ of the events in this group. $77 \%$ of these bursts are originating in the decaying phase of the flare. Average drift rate for type IIs accompanying $\mathrm{C}$ and $\mathrm{M}$ class flares in this category is $0.39 \mathrm{MHz} / \mathrm{s}, 0.31 \mathrm{MHz} / \mathrm{s}$ respectively.

We repeated these calculations by applying Saito's density model (Saito et al, 1977) of the form

$\mathrm{n}(\mathrm{r})=10^{8} \times\left(0.0136 \mathrm{r}^{-2.14}+1.68 \mathrm{r}^{-6.13}\right)$

with a multiplication factor of 10 and obtained almost similar results. 
Histograms representing the distribution of type II parameters such as life time, bandwidth, drift rate, start frequency, shock speed and CME parameters such as CME speed, acceleration/ deceleration corresponding to group I and II are shown in figures $2 \mathrm{a}$ to $2 \mathrm{t}$.

\subsection{Impulsive $X$ class flare events and type II bursts}

$\mathrm{X}$ class flares release large amount of energy. However type II bursts are not found to be associated with these flares in most of the cases. During the period 2002-2015, 70 Impulsive flares of X class are observed with sufficient data. We identified Type IIs and CMEs accompanying the X class flares. Neglecting events with unclear start/end time, insufficient data, our final data set contains $69 \mathrm{X}$ class flares. These flares display sharp rising phase where as the decaying phase is exponential. The details of these flares and the accompanying type IIs \& CMEs are provided in table 4. In 58/69 cases CMEs are temporally associated with flares. In 35/58 cases, the CMEs associated were found to be halo. The speeds of CMEs associated with X class are higher ( avg $1259 \mathrm{~km} / \mathrm{s}$ ) than group I and II counterparts in agreement with results of Magdalenic et al.(2010).In 29/69 events, CME speeds are higher than 1000km/s.

It is interesting to note that only in 9/69 cases type IIs are observed. The characteristics of these 9 type IIs are studied. They possess higher drift rate (avg $0.51 \mathrm{MHz} / \mathrm{s})$. In most of these cases, the associated CMEs are ahead of type IIs supporting the opinion that high pressure blast waves caused by the flares could have been the exciters of type II bursts. However, the correlation between type II speed and CME speed is 0.56 . We find that in $7 / 9$ cases, the type II bursts are originating during rising phase of the flare and their average start frequency, bandwidth and velocity of type II shock waves are significantly higher than group I \& II events. It was observed in few cases that type IIs accompanied C class flares rather than X class flares on a day when both the classes of flares were present.

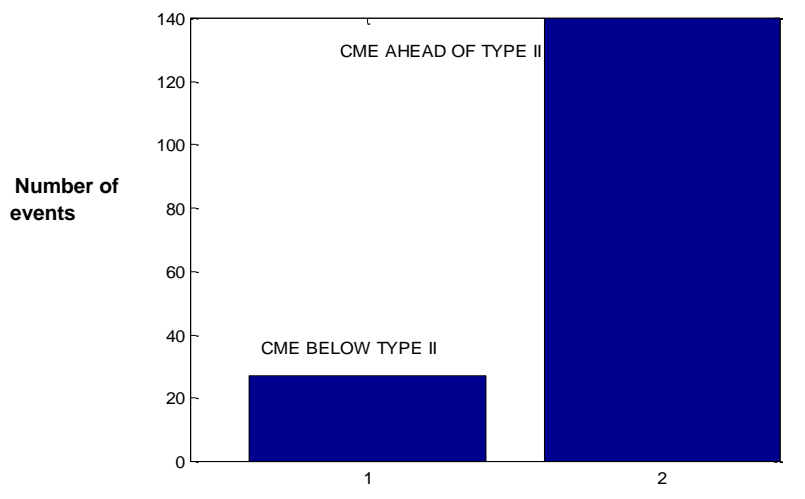

(a)

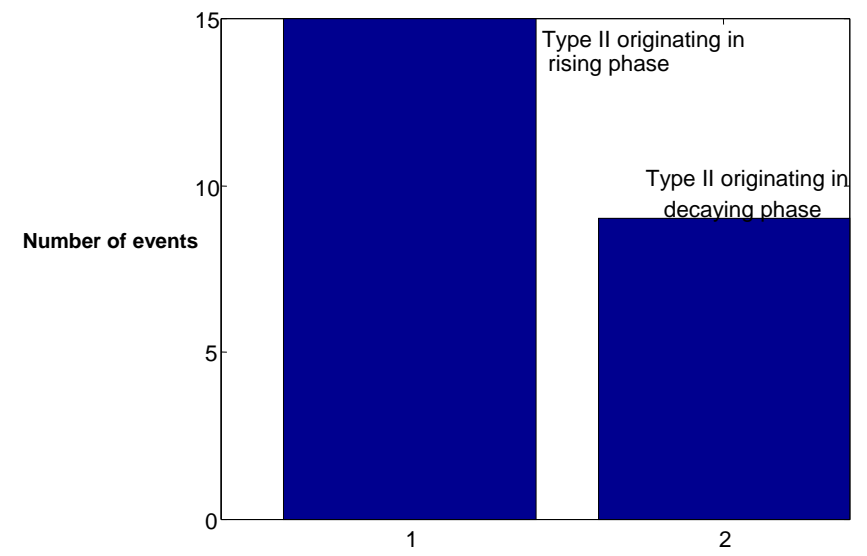

(c)

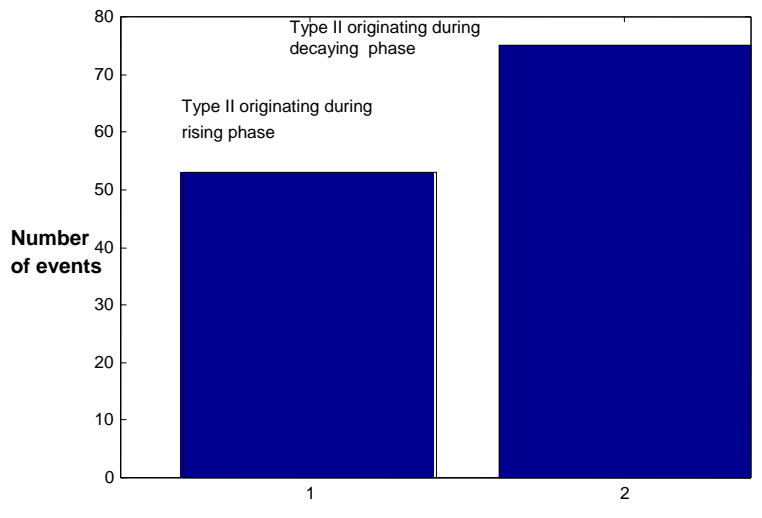

(b)

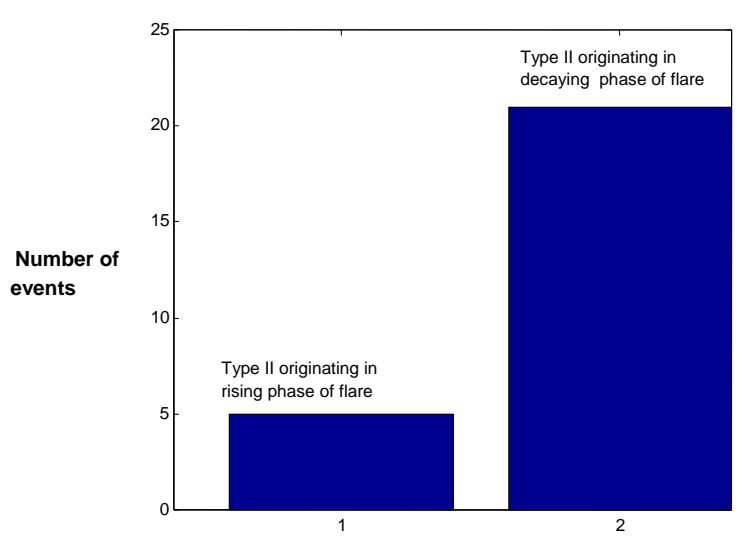

(d) 


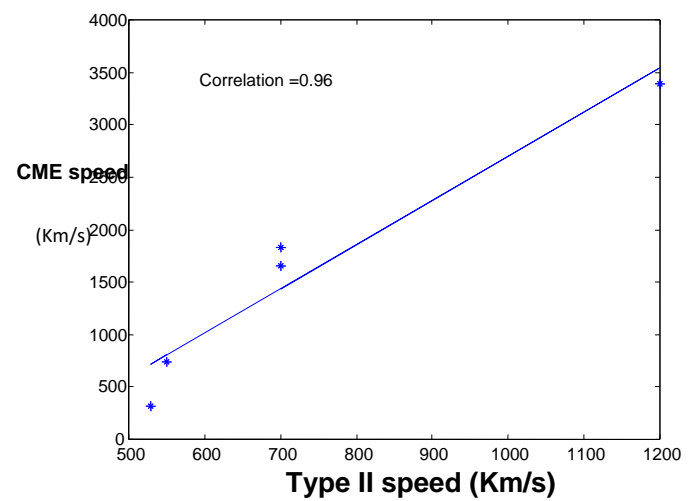

(e)

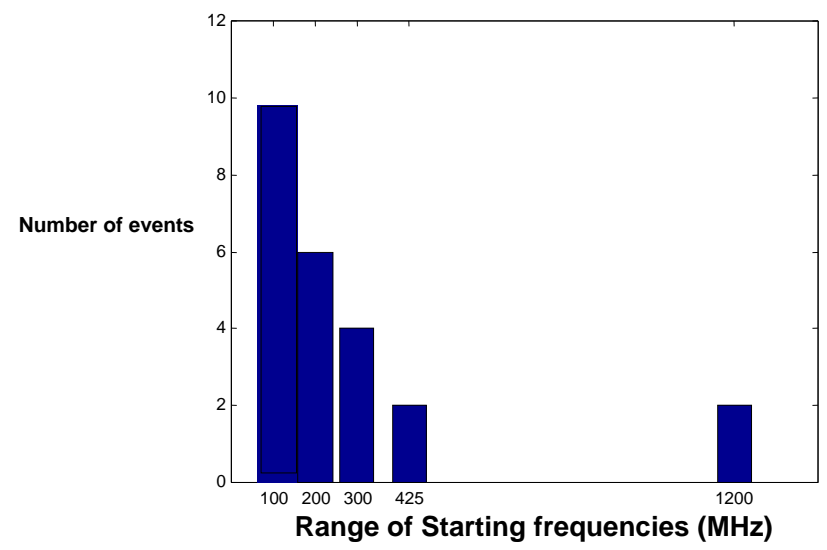

(g)

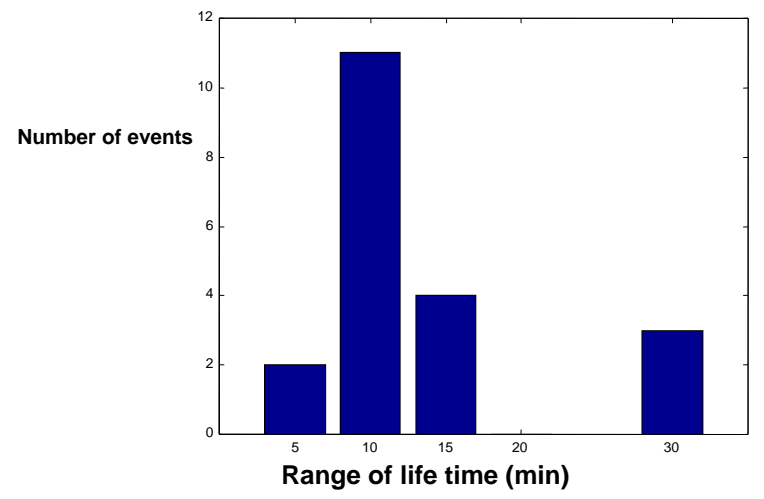

(i)

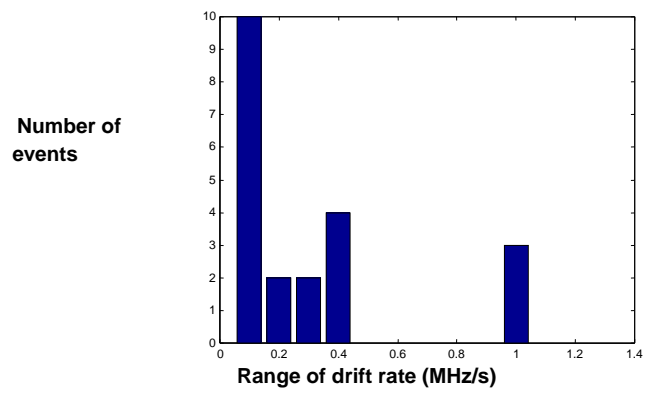

(k)

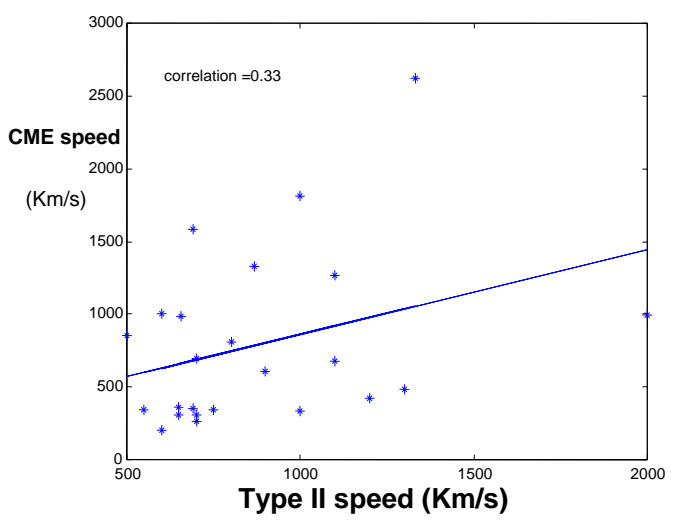

(f)

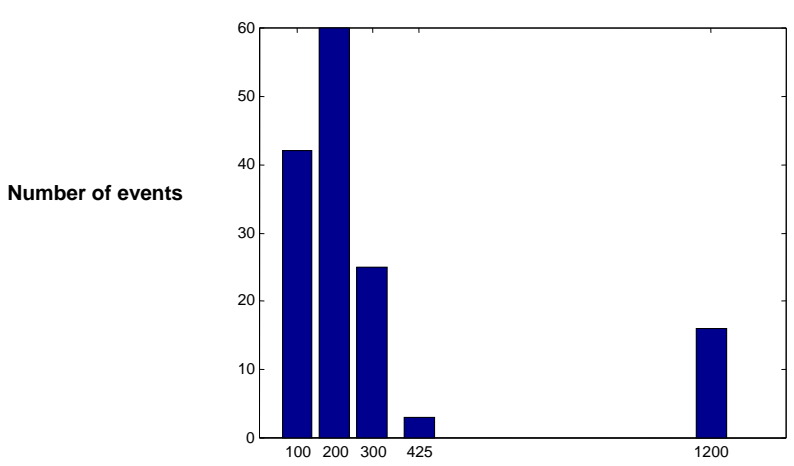

Range of Starting frequencies $(\mathrm{MHz})$

(h)

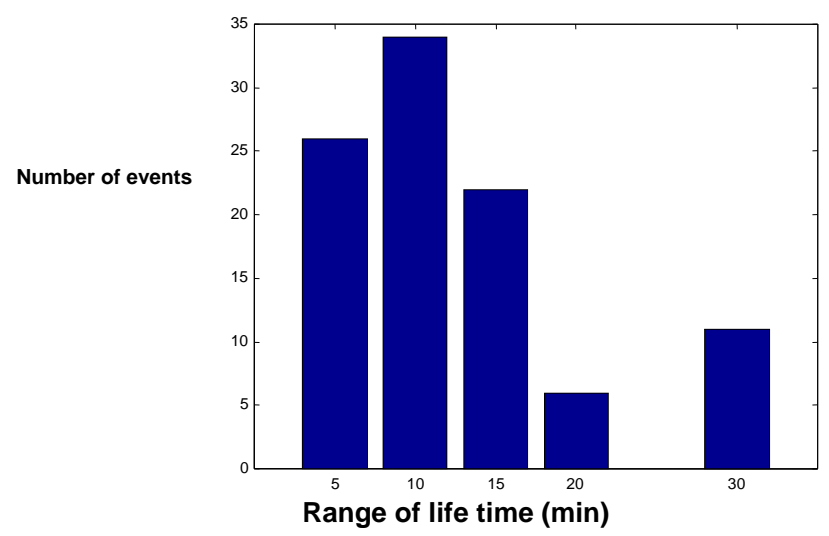

(j)

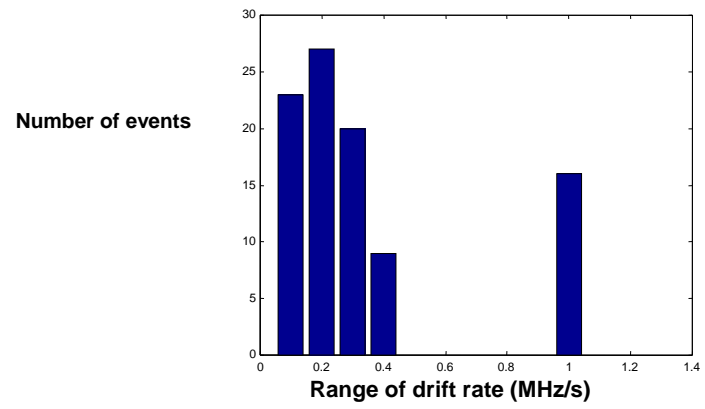

(l) 
Number of events

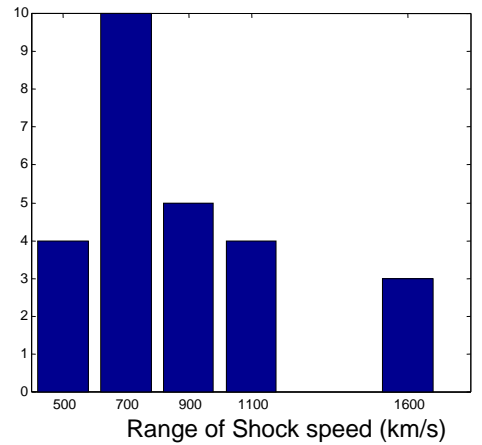

(m)

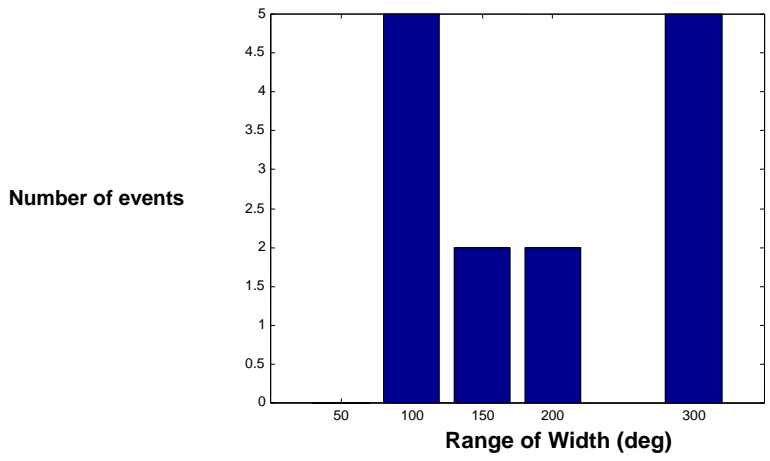

(o)

Number of events

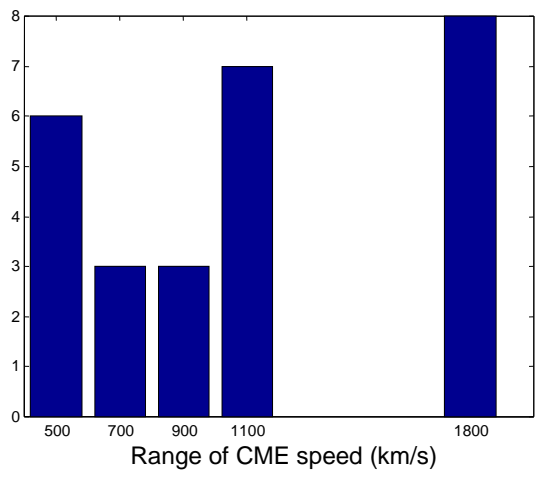

(q)

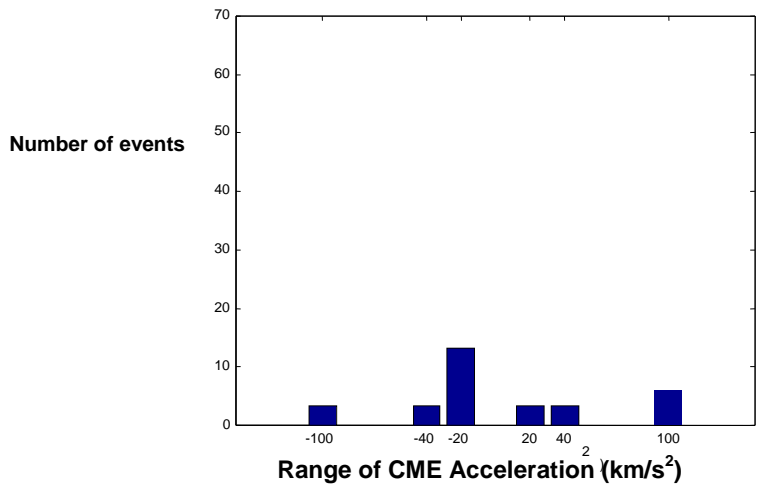

(s)

Figure 2.

a: Distribution of CME based on their height

c:Type II and flare association for group II events

e: Scatter Plot of CME speed and Type II speed for

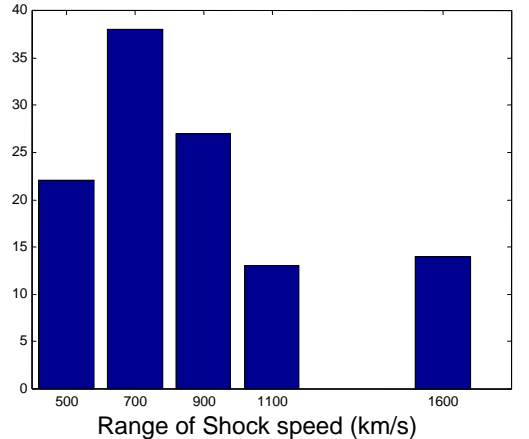

(n)

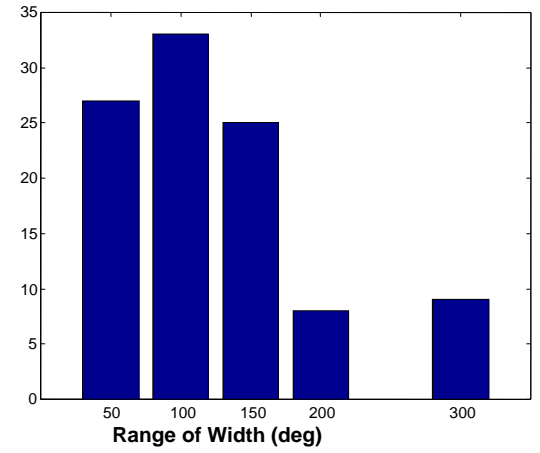

(p)

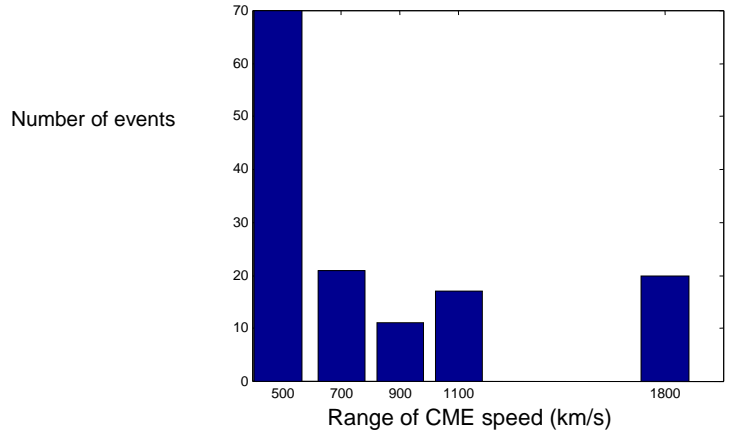

(r)

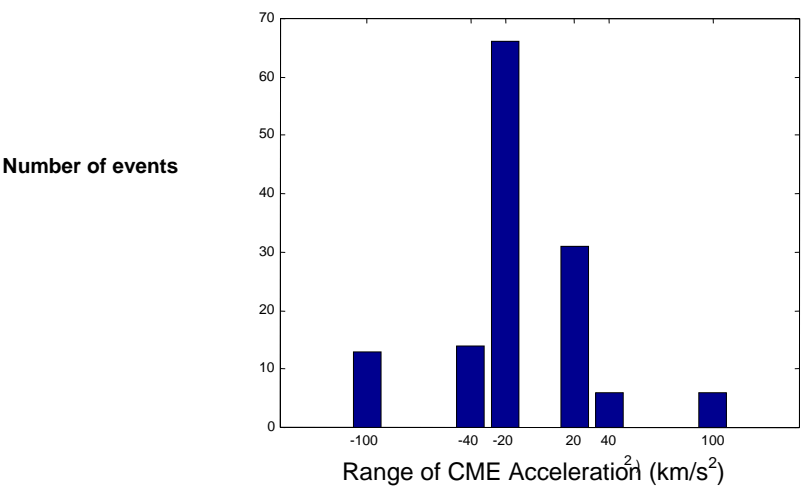

(t)

b: Type II and flare association for group I events

d: Type II and flare association for group III events

f: Scatter Plot of CME speed and Type II speed for Group I 
Groupurnavents University of Shanghai for Science and Technology

ISSN: 1007-6735

g: Distribution of starting frequencies for Group II events h: Distribution of starting frequencies for Group I events

i: Distribution of life time for Group I events

j: Distribution of life time for Group II events

k: Distribution of drift rate for Group II events

I: Distribution of drift rate for Group I events

m:Distribution of shock speed for Group I events

n: Distribution of shock speed for Group II events

o: Distribution of Bandwidth for Group II events

p: Distribution of Bandwidth for Group I events

q: Distribution of CME speed for Group II events

r: Distribution of CME speed for Group I events

s: Distribution of CME acceleration for group II events

$t$ : Distribution of CME acceleration for group II events

Table 1. Type II -Flare - CME parameters for GROUP I events

\begin{tabular}{|c|c|c|c|c|c|c|c|c|c|c|}
\hline \multirow[t]{2}{*}{ Date } & \multicolumn{4}{|c|}{ Type II } & \multicolumn{2}{|c|}{ Flare } & \multirow{2}{*}{$\begin{array}{l}\quad \text { CME } \\
\text { CME } \\
\text { detected } \\
\text { time } \\
\text { (UT) }\end{array}$} & \multicolumn{3}{|c|}{ Height $\left(R_{o}\right)$ based on Newkirk mode } \\
\hline & $\begin{array}{l}\text { start } \\
\text { time } \\
\text { (UT) }\end{array}$ & $\begin{array}{l}\text { End } \\
\text { time } \\
\text { (UT) }\end{array}$ & $\begin{array}{l}\text { Drift } \\
\text { rate } \\
(\mathrm{MHz} / \mathrm{s})\end{array}$ & $\begin{array}{l}\text { Shock } \\
\text { speed } \\
(\mathrm{km} / \mathrm{s})\end{array}$ & $\begin{array}{l}\text { peak } \\
\text { time(UT) }\end{array}$ & Location & & $\begin{array}{l}\text { CME } \\
\text { Height } \\
\text { at the } \\
\text { start of } \\
\text { Type II }\end{array}$ & $\begin{array}{l}\text { Type II } \\
\text { start } \\
\text { height }\end{array}$ & $\begin{array}{l}\text { Type II -CME } \\
\text { height } \\
\text { difference }\end{array}$ \\
\hline $24 / 5 / 2002$ & 0322 & 0328 & 0.2306 & 500 & - & - & 0330 & 1.95 & 1.1488 & -0.8012 \\
\hline $31 / 05 / 2002$ & 0304 & 0314 & 0.0317 & & - & - & 0326 & 2.13 & 1.3375 & -0.7925 \\
\hline $20 / 7 / 2002$ & 2107 & 2137 & 0.0911 & 600 & 2120 & - & 2118 & 6.54 & 1.0392 & -5.5008 \\
\hline $14 / 8 / 2002$ & 0148 & 0209 & 0.0659 & 400 & 0207 & - & 0230 & 2.08 & 1.1488 & -0.9312 \\
\hline $16 / 8 / 2002$ & 0522 & 0554 & 0.0641 & 1700 & - & - & 0530 & 2.71 & 1.0858 & -1.6242 \\
\hline $18 / 8 / 2002$ & 2124 & 2130 & 0.2306 & 1000 & 2150 & - & 2154 & 1.577 & 1.1488 & -0.4282 \\
\hline $21 / 8 / 2002$ & 0154 & 0200 & 0.0639 & 450 & 0144 & - & 0131 & 3.58 & 1.3193 & -2.2607 \\
\hline $22 / 8 / 2002$ & 0156 & 0202 & 0.3417 & 800 & 0152 & - & 0206 & 2.52 & 1.0858 & -1.4342 \\
\hline $24 / 8 / 2002$ & 0106 & 0114 & 0.2188 & 773 & 0113 & - & 0127 & 2.46 & 1.1083 & -1.3517 \\
\hline $24 / 8 / 2002$ & 2051 & 2052 & 0.25 & 718 & 2030 & - & 2020 & 4.797 & 1.1774 & -3.6196 \\
\hline $8 / 9 / 2002$ & 0152 & 0157 & 0.1167 & 900 & - & - & 0206 & 2.06 & 1.2456 & -0.8144 \\
\hline $16 / 9 / 2002$ & 0316 & 0321 & 0.1 & 750 & 0349 & - & 0306 & 3.37 & 1.2793 & -2.0907 \\
\hline $19 / 9 / 2002$ & 0521 & 0524 & 0.4611 & 1000 & 0526 & - & 0554 & 1.61 & 1.1488 & -0.4612 \\
\hline $19 / 9 / 2002$ & 0530 & 0539 & 0.0611 & & - & - & 0554 & 2.09 & 1.2793 & -0.8107 \\
\hline $5 / 10 / 2002$ & 2057 & 2118 & 0.0929 & 272 & 2100 & - & 2154 & 1.21 & 1.0858 & -0.1242 \\
\hline $27 / 10 / 2002$ & 2259 & 2310 & 0.2152 & 900 & 2200 & - & 2318 & 3.87 & 1.1144 & -2.7556 \\
\hline $19 / 10 / 2002$ & 2139 & 2158 & 0.1404 & 850 & 2152 & - & 2206 & 1.68 & 1.0858 & -0.5942 \\
\hline $21 / 1 / 2003$ & 0227 & 0234 & 0.2143 & 700 & 0226 & N14W00 & 0254 & 1.74 & 1.1688 & -0.5712 \\
\hline $23 / 1 / 2003$ & 0448 & 0459 & 0.0894 & 324 & 0449 & S22E12 & 0530 & 1.86 & 1.2394 & -0.6206 \\
\hline $12 / 2 / 2003$ & 0151 & 0156 & 0.4567 & 700 & 0146 & S06W55 & 0230 & 1.32 & 1.0181 & -0.3019 \\
\hline $23 / 4 / 2003$ & 0102 & 0107 & 0.4767 & 679 & 0107 & N18W32 & 0127 & 1.67 & 1.0731 & -0.5969 \\
\hline $25 / 4 / 2003$ & 0530 & 0538 & 0.0667 & 800 & 0544 & N16E61 & 0550 & 1.56 & 1.4497 & -0.1103 \\
\hline $26 / 4 / 2003$ & 2340 & 2347 & 0.3524 & & 2336 & N18W71 & 2326 & 2.76 & 1.078 & -1.682 \\
\hline $29 / 5 / 2003$ & 0106 & 0111 & 0 & 800 & 0102 & S07W46 & 0127 & 4 & 2.1831 & -1.8169 \\
\hline $6 / 6 / 2003$ & 2341 & 2351 & 0.075 & 415 & 2333 & N12E09 & 2354 & 1.85 & 1.3237 & -0.5263 \\
\hline $17 / 6 / 2003$ & 2248 & 2258 & 0.3 & 1000 & 2254 & S07E58 & 2318 & 2.4 & 1.0613 & -1.3387 \\
\hline $17 / 6 / 2003$ & 2003 & 2010 & 0.1667 & 1500 & 1956 & - & 1954 & 5.85 & 1.2793 & -4.5707 \\
\hline $7 / 1 / 2004$ & 0406 & 0420 & 0.0726 & 692 & 0407 & N05E64 & 0406 & 3 & 1.283 & -1.717 \\
\hline $19 / 1 / 2004$ & 2003 & 2014 & 0.2197 & 450 & 1957 & - & 1931 & 3.03 & 1.0858 & -1.9442 \\
\hline $20 / 1 / 2004$ & 0739 & 0750 & 0.2348 & 550 & 0741 & S14W22 & 0830 & 1.42 & 1.0858 & -0.3342 \\
\hline $5 / 4 / 2004$ & 0554 & 0606 & 0.1333 & 615 & 0546 & S16E25 & 0506 & 5.1 & 1.1774 & -3.9226 \\
\hline $16 / 6 / 2004$ & 0423 & 0425 & 0.7917 & 2000 & 0433 & S10E51 & 0436 & 2 & 1.1912 & -0.8088 \\
\hline $5 / 7 / 2004$ & 2232 & 2243 & 0.0848 & 424 & - & - & 2306 & 2.22 & 1.2906 & -0.9294 \\
\hline $24 / 8 / 2004$ & 2110 & 2134 & 0.0535 & 800 & 2107 & - & 2130 & 3.17 & 1.2618 & -1.9082 \\
\hline $31 / 8 / 2004$ & 0541 & 0554 & 0.0987 & 650 & 0533 & N03W88 & 0554 & 2.09 & 1.2456 & -0.8444 \\
\hline $12 / 9 / 2004$ & 0142 & 0148 & 0.1556 & 866 & 0049 & S01W72 & 0036 & 9.82 & 1.3025 & -8.5175 \\
\hline $10 / 10 / 2004$ & 2128 & 2138 & 0.3283 & 559 & 2157 & - & 2154 & 1.52 & 1.0074 & -0.5126 \\
\hline $30 / 10 / 2004$ & 0329 & 0344 & 0.0556 & 800 & 0326 & N14W25 & 0354 & 2.65 & 1.3423 & -1.3077 \\
\hline $30 / 10 / 2004$ & 0613 & 06331 & 0.0351 & 1800 & 0705 & N14W25 & 0654 & 1.87 & 1.3237 & -0.5463 \\
\hline $15 / 1 / 2005$ & 2234 & 2242 & 0.1354 & 1200 & 2257 & N13W03 & 2306 & 1.51 & 1.3065 & -0.2035 \\
\hline
\end{tabular}




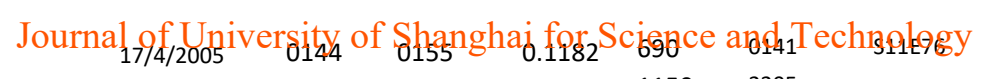

\begin{tabular}{|c|c|c|c|c|c|c|c|c|c|c|}
\hline $17 / 4 / 2005$ & 0144 & $0155^{18}$ & 0.1182 & $690^{\circ}$ & 0141 & ISHE76У & 0206 & 1.83 & 1.196 & -0.634 \\
\hline $19 / 4 / 2005$ & 2150 & 2202 & 0.0861 & 1150 & 2205 & - & 2206 & 1.63 & 1.2984 & -0.331 \\
\hline $2 / 5 / 2005$ & 2239 & 2246 & 0.0357 & & 2241 & - & 2226 & 3.56 & 1.6899 & -1.870 \\
\hline $15 / 5 / 2005$ & 2235 & 2240 & 0.25 & 650 & 2231 & S15E16 & 2326 & 2.26 & 1.2456 & -1.014 \\
\hline $3 / 6 / 2005$ & 0416 & 0429 & 0.1538 & 350 & 0413 & S17E09 & 0332 & 3.98 & 1.1129 & -2.867 \\
\hline $3 / 7 / 2005$ & 0503 & 0510 & 0.3095 & 900 & 0502 & S11E34 & 0530 & 1.61 & 1.1144 & -0.495 \\
\hline $31 / 12 / 2007$ & 0054 & 0058 & 0.1125 & & 0102 & S13E86 & 0142 & 2.05 & 1.3282 & -0.721 \\
\hline $18 / 3 / 2010$ & 2311 & 2314 & 0.4722 & 550 & - & - & 2354 & 1.52 & 1.1912 & -0.328 \\
\hline $25 / 3 / 2010$ & 0128 & 035 & 0.2381 & 850 & & - & 0131 & 3.66 & 1.1488 & -2.511 \\
\hline $12 / 6 / 2010$ & 0058 & 0109 & 0.1864 & 1300 & 0057 & N24W58 & 0131 & 2.18 & 1.0858 & -1.094 \\
\hline $13 / 6 / 2010$ & 0539 & 0545 & 0.3417 & 700 & 0531 & N24W76 & 0530 & 3.35 & 1.0858 & -2.264 \\
\hline $12 / 11 / 2010$ & 0139 & 0146 & 0.3381 & 500 & 0133 & $\mathrm{~S} 22 \mathrm{~W} 10$ & 0224 & 2.585 & 1.1144 & -1.470 \\
\hline $28 / 1 / 2011$ & 0102 & 0111 & 0.1352 & 900 & 0054 & N09W69 & 0125 & 1.81 & 1.1688 & -0.641 \\
\hline $17 / 4 / 2011$ & 2138 & 2144 & 0.175 & 1000 & - & - & 2236 & 2.356 & 1.1912 & -1.164 \\
\hline $8 / 9 / 2011$ & 2158 & 2209 & 0.1212 & - & - & - & 2212 & 1.69 & 1.1688 & -0.521 \\
\hline $23 / 9 / 2011$ & 2353 & 2357 & 0.2833 & 998 & 2210 & N13E59 & 2340 & 3.01 & 1.1646 & -1.845 \\
\hline $2 / 10 / 2011$ & 0051 & 0055 & 0.1792 & 299 & 0044 & N12W25 & 0200 & 1.13 & 1.0952 & -0.034 \\
\hline $15 / 11 / 2011$ & 0012 & 0016 & 0.5417 & 600 & 0000 & - & 0012 & 2.61 & 1.0858 & -1.524 \\
\hline $17 / 11 / 2011$ & 0142 & 0146 & 0.3333 & 600 & - & - & 0200 & 2.251 & 1.1912 & -1.059 \\
\hline $25 / 11 / 2011$ & 2154 & 2159 & 0.1967 & 701 & 2157 & N16W65 & 2224 & 1.71 & 1.2139 & -0.496 \\
\hline $17 / 5 / 2012$ & 0132 & 0142 & 0.2033 & 1100 & 0141 & - & 0148 & 1.45 & 1.1488 & -0.301 \\
\hline $2 / 7 / 2012$ & 0509 & 0515 & 0.4167 & 700 & 0510 & S17E04 & 06 & 1.632 & 1.0731 & -0.558 \\
\hline $6 / 7 / 2012$ & 2309 & 2317 & 0.1375 & 1357 & 2302 & S18W50 & 2324 & 2.68 & 1.2868 & -1.393 \\
\hline $31 / 7 / 2012$ & 0001 & 0010 & 0.1704 & 1000 & 0024 & - & 0012 & 2.482 & 1.2165 & -1.265 \\
\hline $3 / 8 / 2012$ & 0603 & 0616 & 0.0692 & 506 & 0601 & - & 0624 & 2.102 & 1.2757 & -0.826 \\
\hline $6 / 8 / 2012$ & 0443 & 0000 & 0.0882 & 600 & 0437 & S13E75 & 0512 & 1.958 & 1.0858 & -0.872 \\
\hline $8 / 8 / 2012$ & 0059 & 0104 & 0.4 & 657 & 0045 & N20E76 & 0048 & 3.769 & 1.0719 & -2.697 \\
\hline $12 / 8 / 2012$ & 2328 & 2338 & 0.225 & 400 & 2327 & S22E43 & 0054 & 3.25 & 1.0995 & -2.150 \\
\hline $13 / 8 / 2012$ & 0211 & 0215 & 0.0583 & 575 & 0214 & S23E21 & 0224 & 2.742 & 1.536 & -1.206 \\
\hline $7 / 10 / 2012$ & 2120 & 2123 & 0.4 & 1029 & 2122 & - & 2136 & 1.67 & 1.1646 & -0.505 \\
\hline $13 / 5 / 2013$ & 0212 & 0227 & 0.1067 & 1100 & 0212 & N12E81 & 0200 & 3.896 & 1.206 & -2.69 \\
\hline $14 / 5 / 2013$ & 0107 & 0120 & 0.2628 & 1330 & 0124 & N11E63 & 0125 & 1.79 & 1.0216 & -0.768 \\
\hline $6 / 7 / 2013$ & 0156 & 0205 & 0.0963 & 1000 & 0151 & - & 02 & 2.354 & 1.3678 & -0.986 \\
\hline $30 / 8 / 2013$ & 0219 & 0225 & 0.1111 & 700 & 0227 & N11E33 & 0248 & 1.874 & 1.4283 & -0.445 \\
\hline $11 / 10 / 2013$ & 0710 & 0720 & 0.3783 & 924 & 0715 & S27E50 & 0724 & 1.21 & 1.0131 & -0.196 \\
\hline $7 / 11 / 2013$ & 0341 & 0347 & 0.5556 & 600 & 0339 & - & 0424 & 1.096 & 1.0051 & -0.090 \\
\hline $8 / 1 / 2014$ & 0349 & 0402 & 0.1923 & 600 & 0346 & - & 0412 & 1.57 & 1.0858 & -0.484 \\
\hline $17 / 1 / 2014$ & 0228 & 0234 & 0.1111 & 500 & 0222 & - & 0248 & 2.02 & 1.3193 & -0.700 \\
\hline $20 / 2 / 2014$ & 0322 & 0333 & 0.0833 & 600 & 0328 & S11E32 & 0322 & 3.7 & 1.2793 & -2.420 \\
\hline $25 / 2 / 2014$ & 0057 & 0102 & 0.19 & 2000 & 0046 & S12E64 & 0125 & 8.79 & 1.3423 & -7.447 \\
\hline $28 / 2 / 2014$ & 2344 & 2358 & 0.031 & 649 & 2342 & - & 2348 & 2.884 & 1.3678 & -1.516 \\
\hline $17 / 12 / 2014$ & 0443 & 0509 & 0.0333 & 600 & 0442 & - & 05 & 2.395 & 1.3678 & -1.027 \\
\hline $20 / 12 / 2014$ & 0056 & 0111 & 0.0622 & 850 & 0043 & - & 0125 & 1.95 & 1.3472 & -0.602 \\
\hline $9 / 2 / 2015$ & 2314 & 2326 & 0.0903 & 650 & 2325 & N14E51 & 2324 & 1.642 & 1.2793 & -0.362 \\
\hline $3 / 3 / 2015$ & 0134 & 0141 & 0.2857 & 750 & 0133 & - & 0136 & 2.582 & 1.0858 & -1.496 \\
\hline $11 / 3 / 2015$ & 0003 & 0007 & 0.4167 & 700 & 0001 & S16E14 & 0024 & 1.64 & 1.1488 & -0.491 \\
\hline $12 / 3 / 2015$ & 0220 & 0225 & 0.4 & 500 & 0200 & - & 0248 & 2.01 & 1.0995 & -0.910 \\
\hline $17 / 3 / 2015$ & 2332 & 2342 & 0.375 & 600 & 2330 & S21W56 & 2348 & 1.92 & 1.0051 & -0.914 \\
\hline $21 / 4 / 2015$ & 225 & 242 & 0.1284 & 300 & 219 & - & 212 & 3.05 & 1.0925 & -1.957 \\
\hline $22 / 7 / 2015$ & 650 & 708 & 0.1241 & 500 & 659 & S15E20 & 712 & 1.92 & 1.1176 & -0.802 \\
\hline
\end{tabular}

Table 2. Type II -Flare -CME parameters for GROUP II events

\begin{tabular}{|c|c|c|c|c|c|c|c|c|c|c|}
\hline \multirow[b]{2}{*}{ Date } & \multicolumn{4}{|c|}{ TYPE II } & \multicolumn{2}{|c|}{ Flare } & \multirow{2}{*}{$\begin{array}{l}\text { CME } \\
\text { CME } \\
\text { detected } \\
\text { time (UT) }\end{array}$} & \multicolumn{3}{|c|}{ Height $\left(R_{0}\right)$ based on Newkirk model } \\
\hline & $\begin{array}{l}\text { start } \\
\text { time } \\
\text { (UT) }\end{array}$ & $\begin{array}{l}\text { End } \\
\text { time } \\
\text { (UT) }\end{array}$ & $\begin{array}{l}\text { Drift } \\
\text { rate } \\
(\mathrm{MHz} / \mathrm{s})\end{array}$ & $\begin{array}{l}\text { Shock } \\
\text { speed } \\
(\mathrm{Km} / \mathrm{s})\end{array}$ & Peak (UT) & Location & & $\begin{array}{l}\text { CME } \\
\text { Height at } \\
\text { the start of } \\
\text { Type II }\end{array}$ & $\begin{array}{l}\text { Type II } \\
\text { start } \\
\text { height }\end{array}$ & $\begin{array}{l}\text { Type II - } \\
\text { CME } \\
\text { height } \\
\text { difference }\end{array}$ \\
\hline $30 / 5 / 2002$ & 2312 & 2344 & 0.061 & - & - & - & 2326 & 0.9 & 1.0884 & 0.1884 \\
\hline $23 / 8 / 2002$ & 0549 & 0611 & 0.093 & 530 & - & - & 0625 & 1.04 & 1.0858 & 0.0458 \\
\hline
\end{tabular}




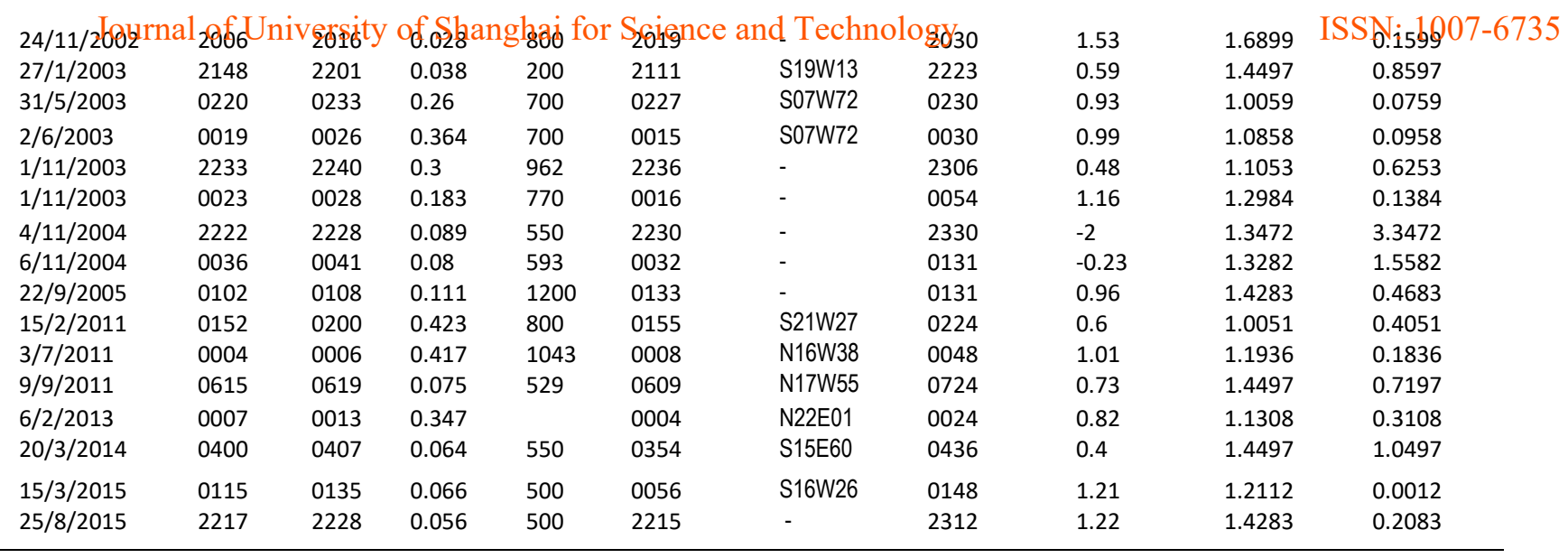

Table 3. Type II -Flare parameters for GROUP III events

\begin{tabular}{|c|c|c|c|c|c|c|c|}
\hline \multirow[b]{2}{*}{ Date } & \multicolumn{4}{|c|}{ Type II } & \multicolumn{2}{|c|}{ Flare } & \multirow{2}{*}{$\begin{array}{l}\text { Type II height } \\
\left(R_{\circ}\right) \text { based on } \\
\text { Newkirk model }\end{array}$} \\
\hline & $\begin{array}{l}\text { start time } \\
\text { (UT) }\end{array}$ & $\begin{array}{l}\text { End time } \\
\text { (UT) }\end{array}$ & $\begin{array}{l}\text { Drift rate } \\
(\mathrm{MHz} / \mathrm{s})\end{array}$ & $\begin{array}{l}\text { Shock speed } \\
(\mathrm{Km} / \mathrm{s})\end{array}$ & $\begin{array}{l}\text { Peak } \\
\text { (UT) }\end{array}$ & Location & \\
\hline $16 / 7 / 2002$ & 2201 & 2206 & 0.26 & 557 & 2141 & - & 1.1507 \\
\hline $19 / 7 / 2002$ & 0331 & 0333 & 0.24 & 325 & 0246 & - & 1.1414 \\
\hline $25 / 8 / 2002$ & 0325 & 0329 & 0.23 & 484 & 0323 & - & 1.1053 \\
\hline 2/9/2002 & 0012 & 0014 & 0.79 & 1897 & ..... & - & 1.116 \\
\hline 8/9/2002 & 0156 & 0203 & 0.07 & 500 & 0136 & - & 1.2686 \\
\hline $30 / 9 / 2002$ & 0425 & 0428 & 0.11 & 1000 & 0420 & - & 1.4811 \\
\hline $14 / 10 / 2002$ & 0003 & 0008 & 0.31 & 1000 & 0005 & - & 1.1912 \\
\hline $31 / 1 / 2003$ & 2208 & 2226 & 0.07 & 505 & 2241 & - & 1.2086 \\
\hline $19 / 7 / 2003$ & 0139 & 0143 & 0.06 & 551 & 0136 & N16W20 & 1.4982 \\
\hline $20 / 1 / 2004$ & 0748 & 0757 & 0.12 & 850 & 0741 & S14W22 & 1.2456 \\
\hline $27 / 4 / 2004$ & 0716 & 0735 & 0.14 & 600 & 0723 & - & 1.0731 \\
\hline $24 / 6 / 2004$ & 0619 & 0626 & 0.24 & 565 & 0605 & S11W53 & 1.1507 \\
\hline $31 / 8 / 2004$ & 0547 & 0554 & 0.13 & 400 & 0536 & N03W88 & 1.2984 \\
\hline $18 / 8 / 2006$ & 2357 & 2359 & 0.75 & 630 & 2326 & - & 1.1488 \\
\hline $19 / 2 / 2007$ & 0018 & 0024 & 0.16 & 500 & 0013 & S12E04 & 1.2793 \\
\hline 3/3/2011 & 0343 & 0350 & 0.24 & 900 & & - & 1.1144 \\
\hline 30/9/2011 & 0252 & 0256 & 0.07 & 676 & 0249 & $\mathrm{~N} 13 \mathrm{~W} 29$ & 1.4352 \\
\hline $13 / 12 / 2011$ & 0316 & 0323 & 0.15 & - & - & - & 1.1912 \\
\hline $15 / 12 / 2013$ & 2027 & 2042 & 0.09 & 600 & - & - & 1.2165 \\
\hline 22/6/2015 & 0600 & 0607 & 0.07 & 325 & 0518 & - & 1.2305 \\
\hline
\end{tabular}

Table 4. X class flare - Type II - CME parameters

\begin{tabular}{|c|c|c|c|c|c|c|c|c|}
\hline \multirow[t]{2}{*}{ Date } & \multicolumn{3}{|c|}{ Flare } & \multicolumn{3}{|c|}{ Type II } & \multicolumn{2}{|c|}{ CME } \\
\hline & $\begin{array}{l}\text { peak time } \\
\text { (UT) }\end{array}$ & Location & class & $\begin{array}{l}\text { start } \\
\text { time(UT) }\end{array}$ & $\begin{array}{l}\text { end } \\
\text { time(UT) }\end{array}$ & $\begin{array}{l}\text { drift } \\
\text { date(MHz/s) }\end{array}$ & $\begin{array}{l}\text { Detected } \\
\text { time (UT) }\end{array}$ & $\begin{array}{l}\text { Speed } \\
(\mathrm{Km} / \mathrm{s})\end{array}$ \\
\hline $27 / 5 / 2003$ & 0028 & S07 W20 & $\mathrm{X} 1.3$ & 2302 & 2310 & 0.52 & $\ldots$ & \\
\hline $29 / 5 / 2003$ & 0057 & S07W46 & $\mathrm{X} 1$ & $\begin{array}{l}\text { Insufficient } \\
\text { data }\end{array}$ & & & 0127 & 1237 \\
\hline $9 / 6 / 2003$ & 2139 & N12W32 & $\mathrm{X} 1.7$ & 2138 & 2152 & 0.392 & $\ldots$ & \\
\hline $10 / 6 / 2003$ & 0002 & N12W36 & $\mathrm{X} 1.4$ & & & & 0056 & 730 \\
\hline $15 / 6 / 2003$ & 2356 & S07E85 & $\mathrm{X} 1.3$ & & & & 2354 & 2053 \\
\hline $19 / 10 / 2003$ & 1649 & N06E53 & $\mathrm{X} 1.1$ & & & & 1708 & 472 \\
\hline $23 / 10 / 2003$ & 0826 & S16E70 & X5.4 & & & & 0854 & 511 \\
\hline
\end{tabular}




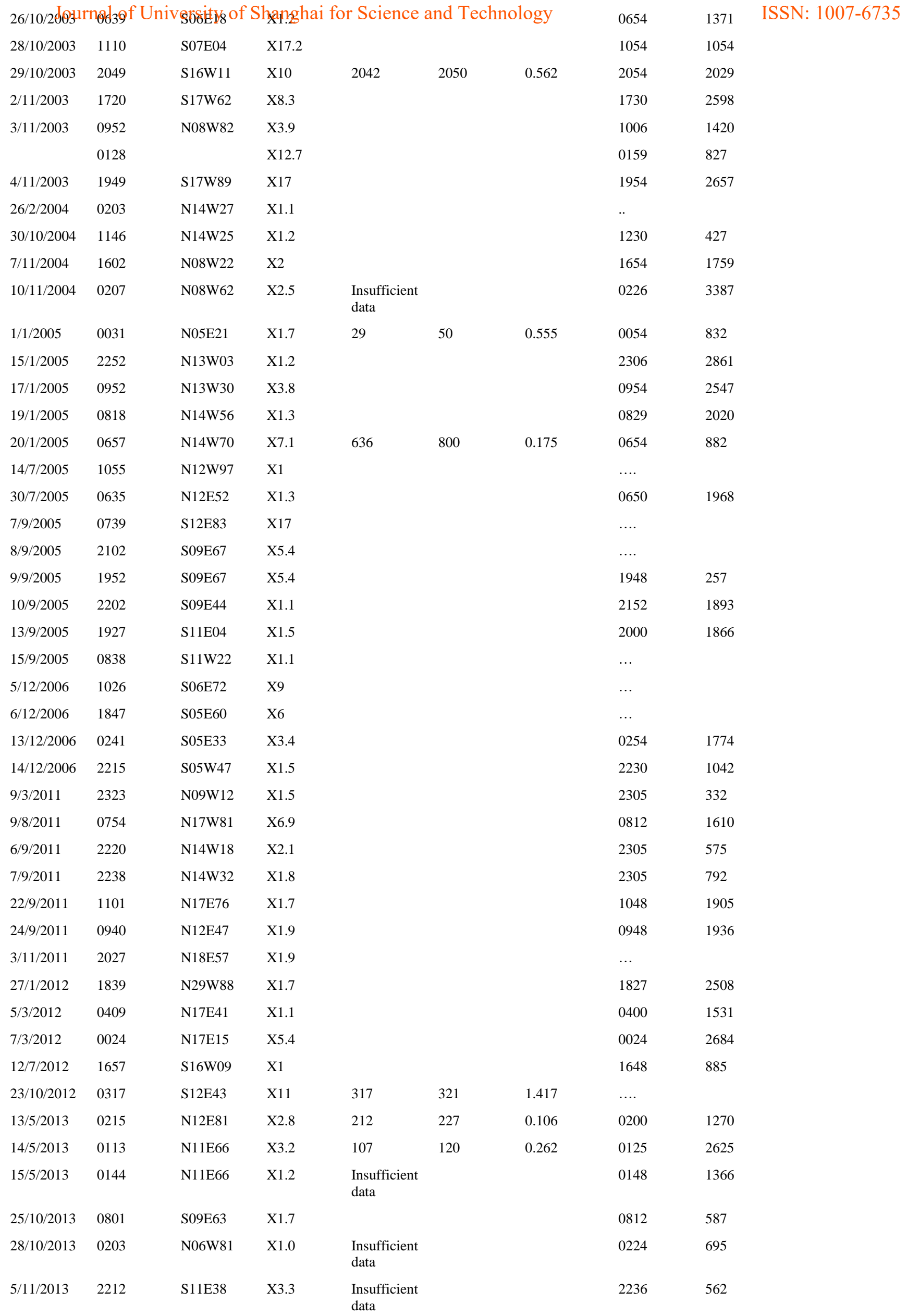




\begin{tabular}{|c|c|c|c|c|c|}
\hline $10 / 11 / 2013$ & 0514 & S11W29 & $\mathrm{X} 1.1$ & 0536 & 682 \\
\hline $29 / 3 / 2014$ & 1748 & N10W36 & $\mathrm{X} 1$ & 1812 & 528 \\
\hline $25 / 4 / 2014$ & 0024 & S14W90 & $\mathrm{X} 1.7$ & 0048 & 456 \\
\hline \multirow[t]{2}{*}{$10 / 6 / 2014$} & 1252 & S17E82 & $\mathrm{X} 1.5$ & 1310 & 1469 \\
\hline & 1142 & S15E80 & $\mathrm{X} 2.2$ & 1148 & 924 \\
\hline $11 / 6 / 2014$ & 0906 & X18E65 & $\mathrm{X} 1$ & 0924 & 829 \\
\hline $19 / 10 / 2014$ & 0503 & S13E57 & $\mathrm{X} 1.3$ & 0448 & 139 \\
\hline $22 / 10 / 2014$ & 1428 & S14E13 & X1.6 & 1400 & 655 \\
\hline $24 / 10 / 2014$ & 2141 & S12W21 & X3.1 & 2148 & 184 \\
\hline $25 / 10 / 2014$ & 1708 & S16W21 & $\mathrm{X} 1$ & 1736 & 171 \\
\hline $26 / 10 / 2014$ & 1056 & S18W40 & $\mathrm{X} 2$ & $\ldots$. & \\
\hline $27 / 10 / 2014$ & 1447 & S17W52 & $\mathrm{X} 2$ & 1512 & 170 \\
\hline $7 / 11 / 2014$ & 1724 & N17E40 & X1.6 & 1712 & 469 \\
\hline $11 / 3 / 2015$ & 1622 & S17E02 & $\mathrm{X} 2.1$ & 1700 & 240 \\
\hline $5 / 5 / 2015$ & 2211 & N12E70 & $\mathrm{X} 2.7$ & 2224 & 715 \\
\hline
\end{tabular}

\section{CONCLUSIONS}

In this paper we study the relationship between type II bursts and CMEs. We find that in the metric domain in $70 \%$ of the events in our data set, the type II formation height is below the CME height indicating that CMEs are less successful in exciting type II bursts in the metric domain. The main results of the paper are summarized as follows.

1. We apply the Newkirk density model to determine the type II bursts and compare it with CME height at the onset of type II.

2. We classify the events as group I, II \& III depending whether type height - CME height is positive, negative or CME is not present respectively. We have found that type II parameters are slightly higher for group I events than group II events. Type II bursts not associated with CMEs possess lesser shock speed, bandwidth and duration. In $83 \%$ of type II events, our results suggest that inspite of temporal association, majority of the CME driven shocks are not successful in exciting type II bursts in 35-450 MHz domain.

3. CME parameters are greater for group II events than group I events. This supports the earlier results which indicated that CMEs successful in exciting type II bursts are stronger with greater speed \& width.

4. Fairly equal amount of flare association exists in group I, II \& III events. Despite poor correlation is observed between type IIs and $\mathrm{X}$ class flares, such type IIs possess higher drift rates. Majority of X class flares are accompanied by CMEs which possess higher speed (avg $1259 \mathrm{~km} / \mathrm{s})$.

5. The type II bursts likely to have been excited by CMEs are originating during the rising phase of the flares in majority of the events and in case of type II bursts supposedly not excited by the CMEs, majority of them are originating in the decaying phase of flares.

6. We applied Saito (1977) density model and obtained similar results.

\section{ACKNOWLEDGEMENTS}

We are deeply indebted to authorities of Indian Institute of Astrophysics, Bangalore, CMR Institute of Technology, Bangalore and Bangalore university, Bangalore for the support and encouragement. We are grateful to the authorities of Culgoora observatory, SOHO and RHESSI teams for their open data policy.

\section{REFERENCES}

1. Aurass, H., 1997, Coronal Physics from Radio and Space Observations, edited by G. Trottet, Springer, Berlin, p.135, 1997.

2. Bemporad, A., Poletto, G., Suess, S. T., Ko, Y. K., Parenti,S., Piley,P., Romoli, M., \& Zurbuchen, T. Z. 2003, ApJ, 593, 1146

3. Brueckner, G. E, et al. 1995, Sol. Phys., 162, 357

4. $\quad$ Cane, H. V., Sheeley, N. R., Jr., and Howard, R. A.: 1987, J. Geophys. Res. 92, 9869.

5. Chen, P.F.: Living Reviews in Solar Physics, Volume 8, Issue 1, article id.1, 92 pp

6. Cho, K.-S., Lee, J., Gary, D. E., Moon, Y.-J., \& Park, Y. D. 2007, ApJ, 665,799

7. Cho, K.S., et al. 2011, Astronomy \& Astrophysics, Volume 530, id.A16, 5 pp

8. $\quad$ Cho, K.-S., et al. 2005, J. Geophys. Res., 110, A12101

9. Cho, K.-S., et al. 2008, A\&A, 491, 873

10. Cho, K.-S.,et al, Lee, J., Gary, D. E., Moon, Y.-J., \& Park, Y. D. 2007, ApJ, 665,799

11. Cho, K-.S. ,et al, 2013, Solar Physics, Volume 284, Issue 1, pp.105-127 
13. Cliver, E. W., Kahler, S. W., \& Reames, D. V. 2004, ApJ, 605, 902

14. Dulk, G. A., 1970, Proceedings of the Astronomical Society of Australia, Vol. 1, p.308

15. Gary et al. 1984, A\& A.134, 222

16. Gergely,T.E., et al. 1983; Astrophysical Journal, 268, 403-411.

17. Giacalone,J.:Jokipii,J.R.,2005,American Geophysical Union, Fall Meeting, abstract \#SH52A-03

18. Gopalswamy, N. and Kundu, M. R.: 1992, in G. P. Zank and T. K. Gaisser (eds.), AIP Conf. Proc.

19. Gopalswamy \& S. Yashiro, 2011,Astrophys. J., 736, L17

20. Gopalswamy, N., 2006, Planetary Space Sci, 52, 1399-1413, 2004a

21. Gopalswamy, N. (2000), Type II radio bursts, in Radio Astronomy at Long Wavelengths, Geophys. Monogr. Ser., vol. 119, edited by R. G. Stone etal., p. 123, AGU, Washington, D. C.

22. Gopalswamy et al. 2001, Journal of Geophysical Research, Volume 106, Issue A12, p. 29219-29230

23. Gopalswamy et al. 2009a; Gopalswamy, et al., Sol. Phys., 259, 227-254

24. Guhathakurta, M.; Fisher, R.; Strong, K.,1998; Observational Plasma Astrophysics : Five Years of Yohkoh

and

Beyond. Edited by Tetsuya Watanabe, Kluwer Academic Publishers, v. 229., p.13

25. Kosugi, T., 1971, Solar Phys., 48, $339-356$.

26. Lara, A., Gopalswamy, N. Nunes, S., Munoz, G., \& Yashiro, S. 2003, Geophys. Res. Lett., $30(12), 801627$. Y., Dulk, G. A., Vourlidas, A., \& Bougeret, J.-L. 2001,J. Geophys. Res., 160, 25,301

28. Lin, 2002,In: Solar variability: from core to outer frontiers. Ed. A. Wilson. ESA SP-506, Vol. $2 . \quad$ Noordwijk: ESA Publications Division, ISBN 92- 9092-816-6, 1035 - 1044

29. Liu, Y., Luhmann, J.G., Bale, S.D., Lin, R.D.: 2009, Astrophys. J. Lett. 691, L151.

30. Magdalenic et al, 2010,The Astrophysical Journal,

31. Magdalenic et al , 2012, The Astrophysical Journal,

32. Magdalenic et al,2008, Solar Physics, Volume 253,

33. Maia, D., Pick, M., Vourlidas, A., \& Howard, R. 2000, ApJ, 528, L49

Volume 718, Issue 1, pp. 266-278

Volume 746, Issue 2, article id. 152, 8 pp

Issue 1-2, pp. 305-317

34. Mancuso, S., et al, Astronomy and Astrophysics,

Volume 463, Issue 3, March I 2007, pp.1137-114133.

35. Mann, G. 1995, J. Plasma Phys., 53, 109

36. Mann, G., et al,2007, The High Energy Solar Corona:

Volume 725. Springer-Verlag Berlin Heidelberg, p. 203

37. Moses, D., Clette, F., Delaboudinière, J.P., Artzner, G.E., et al.: 1997, Solar Phys. 175,571 - 599.

38. Miteva, Mann, 2007, Astronomy and Astrophysics, Volume 474, Issue 2, November I 2007, pp.617-625

39. Nelson, G. J. and Robinson R. D.: 1975, Proc.

Astron. Soc. Aust. 2, 370.

40. Nelson, G.J., and Melrose, D.B., in Solar Radio

Physics, edited by D.J.McLean and R.D.Robinson, Cambridge,

Newyork, p 333, 1985.

41. Newkirk, G. A.: 1961, Astrophys. J. 133, 983.

42. Nindos,A.;Alissandrakis,C.E.;Hillaris,A.;Preka-

id.A31, $12 \mathrm{pp}$

Papadema,P.,2011, Astronomy \& Astrophysics, Volume

531,

43. Parenti,s.: et al, 2000, Astronomy and Astrophysics, v.363, p.800-814

44. Pick, M., \& Vilmer, N. 2008, A\&AR, 16, 1

45. Prakash et al. 2010, Solar Physics, Volume 266,

46. Ramesh et al. 2010, The Astrophysical Journal,

47. Reiner, M. J., Kaiser, M. L., Gopalswamy, N.,

Geophys Res. 106, 25279-25290

48. Reiner, M. J., M. L. Kaiser, and J. L. Bougeret

49.

Saito, K., Poland, A. I., \& Munro, R. H. 1977, Sol.

50. Schwartz, et al, 2011, Physical Review Letters, vol.

51. Sckopke, N.1995, Advances in Space Research

Issue 1, pp.135-147

Volume 712, Issue 1, pp. 188-193

Aurass, H., Mann, G., Vourlidas, A., Maksimovic, M., 2001a. J.

, 2001b, J. Geophys. Res., 106, 29,989.

Phys. 55, 121

107, Issue 21, id. 215002

(ISSN 0273-1177), vol. 15, no. 8-9, p. 261-269

52. Stewart, R. T., M. McCabe, M. J. Koomen, R. T.

Hansen, G. A. Dulk, Solar Phys., 36, 203, 1974a

53. Stewart, R. T., R. A. Howard, F. Hansen, T. Gergely, M. Kundu, Solar Phys., 36, 219, 1974b

54. Subramanian \& E.Ebenezer, 2006,A\&A,683,451

55. Thompson, B.J., Plunkett, S.P., Gurman, J.B.,

Res. Lett. 25, 2465.

56. Thomsen ey al, 1985, Journal of Geophysical Research (ISSN 0148-0227), 90, 137-148

57. Vršnak, B., Cliver, E.: 2008, Solar Phys. 253, 215.

58. Vřsnak, B., Aurass, H., Magdaleni'c, J., \& Gopalswamy, N. 2001,A\&A, 377, 321

59. Wagner, W. J. \& MacQueen, R. M., 1983, A\&A, 120,136

60. Wild, J.P., and McReady, L.L. 1950, Aust.J. Sci.Res, A3, 387

61. Zhang, M., \& Low, B. C. 2001, ApJ, 561, 406

62. Zheleznyakov, V. V.: 1970, Radio Emission of the Sun and the Planets. Oxford: Pergamon Press Ltd. 